\title{
Warum soll Lernen Spaß machen?
}

\section{Eine dispositivanalytische Untersuchung schulischen Lernens}

\author{
Michael Brandmayr
}

Angenommen: 21. Mai 2016 / Online publiziert: 4. Juli 2016

(C) Der/die Autor(en) 2016. Dieser Artikel ist eine Open-Access-Publikation.

Zusammenfassung In den vergangenen Jahren hat die Zahl jener Publikationen zugenommen, die sich um eine Zueignung des Lernbegriffs in das pädagogische Vokabular bemühen. Während in der bildungswissenschaftlichen Rezeption Beiträge von benachbarten Disziplinen wie der Lernpsychologie und der Neurobiologie berücksichtigt werden, trifft das auf soziologische und bildungssoziologische Beiträge nur in geringem Ausmaß zu. Gesellschaftliche Deutungsweisen und Diskurse über pädagogische Leitbilder idealen Lernens wurden bislang kaum untersucht, gleichwohl ihr Bezug zur Praxis in pädagogischen Institutionen naheliegend ist.

Der folgende Beitrag soll eine alternative Perspektive aufzeigen, die für einen pädagogischen Blick auf das Lernen relevant sein kann. Unter Rückgriff auf Annahmen der wissenssoziologische Diskursanalyse soll argumentiert werden, dass ein diskursiv formiertes Leitbild von idealem Lernen den handelnden Akteur/innen im Bildungsbereich ein Wissen bereitstellt, welches als professionsspezifisch legitimiert gilt. Darin enthaltene Deutungsmuster werden im Bildungsdispositiv neu formiert und können in der Praxis als handlungsanleitende Maßstäbe fungieren. Sie verfügen somit über zweierlei normierende Wirkung, nämlich indem sie einerseits als Normative Einfluss auf die schulische Praxis von Lernprozessen nehmen und andererseits durch ihre spezifische Praxisform Positionen von Subjektivität bereitstellen. Dies zeigt der Beitrag anhand der Analyse des Diskurses um „Freude am Lernen“.

Schlüsselwörter Lernen · Diskursanalyse $\cdot$ Freude am Lernen · Hidden Curriculum $\cdot$ Lerndiskurs

\footnotetext{
Mag. M. Brandmayr $(\square)$

School of Education, Institut für LehrerInnenbildung und Schulforschung, Universität Innsbruck, Innrain 52d, 6020 Innsbruck, Österreich E-Mail: Michael.brandmayr@uibk.ac.at
} 


\section{Why should learning be fun?}

Analysing the dispositif underlying learning arrangements in schools

Abstract The last years have seen a rise in the number of publications which promote a pedagogic view on the idea of learning (cf. Schrittesser 2012). In contrast to arguments of the psychology of learning and neurosciences, there has been little reception of sociological arguments within the pedagogic discourse so far. Despite the self-evident relevance for pedagogic actions, little attentions has been paid to discourses of guiding conceptions of ideal learning and the dominant interpretative schemes within them.

This paper emphazises the relevance of sociological arguments for a pedagogic perspective on learning processes. Following assumptions of the Sociology of Knowledge Approach to Discourse, it argues that discourses produce legitimate knowledge about ideal forms of learning. The dispositif deriving from this knowledge provides actors with frameworks of interpretation and structures learning processes in pedagogic institutions. It also offers positions of subjectivity. The knowledge itself commands a guiding function for stakeholders by certain implied interpretations on ideal learning. It shall be concluded that discourses in society have two effects of normalizing the discursive field, by on the one hand shaping current forms of learning in pedagogic institutions and on the other hand transmitting certain forms of subjectivity to stakeholders. An analysis of the discourse on "making learning fun" in Austria illustrates this argument.

Keywords Learning $\cdot$ Discourse analysis $\cdot$ Making learning fun $\cdot$ Hidden Curriculum $\cdot$ Discourse of learning

\section{Diskurstheorie und schulisches Lernen}

Das Thema Lernen hat in pädagogischen Diskussionen in jüngerer Zeit zunehmende Beachtung gefunden, gleichwohl (bildungs-)soziologische Beiträge zu Fragen des schulischen Lernens bislang gering rezipiert wurden. Der folgende Beitrag möchte diese Perspektive aufgreifen und schließt dabei an den Ansatz der subjektwissenschaftlichen Lerntheorie an (vgl. Holzkamp 1992, 1993; Ludwig 2005). Im Anschluss an Holzkamps Unterscheidung in expansives und defensives Lernen richtet sich dort der Fokus auf schulische Strukturen und Verfahrensweisen, die Einfluss auf den Charakter von Lernverläufen nehmen. Lernen stellt ,eine individuell-kognitive Bedingung für Bildung dar" (Ludwig 2005, S. 329), während das Subjekt aber „auch Produkt gesellschaftlicher Strukturen, in diese verstrickt und ihnen unterworfen" (Grotlüschen 2014, S. 231) ist. In diesem widersprüchlichen Verhältnis befindet sich das Lernsubjekt der Schule, das zwar Räume selbstbestimmten Lernens vorfinden kann, aber durch den gesellschaftlichen Wunsch zur administrativen Planbarkeit von Lernprozessen zahlreichen Normierungen unterliegt.

Die Frage, welche Prinzipien oder Deutungsmuster bei der Konstitution schulischer Lernprozesse relevant sein können, wird in der subjektwissenschaftlichen Lerntheorie eher oberflächlich angesprochen. Holzkamp (1992, S. 14) stellt fest, dass 
die Schule „,durch die Art der Unterrichtsorganisation [...] etwaige eigene sachbezogene expansive Lernbemühungen der Schülerinnen/Schüler permanent behindert und stört", und nennt als solche organisationalen Elemente beispielsweise die 45Minuten-Taktung, Vorstellungen von Schuldisziplin oder den repetitiven Charakter von Prüfungen. Diese Elemente führt Holzkamp wiederum auf die Notwendigkeit der administrativen Plan- und Überprüfbarkeit von Lernprozessen zurück, die er (bildungssoziologisch) als Folge der Qualifikations- und Allokationsfunktion von Schule betrachtet.

Entwicklungen von Unterricht, wie die Zunahme offener Unterrichtsformen (vgl. Brügelmann 2008), offenbaren jedoch einen Schwachpunkt dieser strukturfunktionalistischen Argumentation: Gegenwärtige Unterrichtsformen kommen vielfach ohne feste Zeitstrukturen, mit (scheinbar) flacheren Hierarchien (Stichwort: Lehrer/in als Coach) aus und bieten Schüler/innen mehr Gestaltungsmöglichkeiten; dies sollte implizieren, dass die Schule (im Sinne Holzkamps interpretiert) expansive Lernbestrebungen vermehrt ermöglichen, die ,administrative Planbarkeit“ des schulischen Lernens sinken würde, Lernprozesse weniger vergleichbar, Schüler/innen in der Gestaltung freier sein müssten - doch das alles scheint fraglich. Nicht zuletzt weisen etwa Patzner (2007) und Gruschka (2011) darauf hin, dass die Intention hinter diesen Entwicklungen tatsächlich nicht primär in der Steigerung von Autonomie und selbstbestimmten Bildungsprozessen im Unterricht bestünde. Dies führt zu der Überlegung, die offene Frage nach Genese, strategischen Intentionen sowie möglichen Konsequenzen hinter den Veränderungen schulischer Praxisformen unter diskursanalytischen Perspektiven zu betrachten.

Ein diskurstheoretischer Zugang ermöglicht indess, Prinzipien und Deutungsmuster hinter schulischen Lernprozessen zu analysieren, die allgemein im Kontext eines bildungspolitischen und schulpädagogischen Diskurses um ein neues Leitbild von Schule und Unterricht existieren und sich in Aussagen manifestieren. Der Beitrag verfolgt das Ziel einer Rekonstruktion und Kritik dieser Deutungen und beschreibt schulisches Lernen daher als eine diskursiv formierte soziale Praxis. In der diskursiven Auseinandersetzung wird festgelegt, was als effektive Verfahren und Methoden des Lernens, wünschenswerte Ziele, notwendige Bedingungen (wie „Spaß“ oder „Freiraum“), strukturelle Voraussetzungen in Institutionen (wie „Individualisierung“) u. v. m. gelten. Diskurse setzen dem Handeln der Individuen Grenzen; sie definieren, was legitim gesagt und getan werden kann bzw. was nicht. Anders als die subjektwissenschaftliche Theorie soll nicht beansprucht werden, Erkenntnisse auf der Ebene individueller, konkreter Lernvollzüge zu formulieren. Dennoch wird argumentiert, dass aus der Beziehung zwischen dem Diskurs und dem Praxisfeld Schule Einflüsse auf der Ebene individueller Lernhandlungen entstehen, und zwar aus drei Gründen:

- Diskurse produzieren Wissen um einen Gegenstand. Die Institutionalisierung dieses Wissens in Dispositiven führt zur Tradierung und Legitimation von spezifischen Handlungsweisen. Das Bildungsdispositiv greift auf Basis des diskursiv erzeugten Wissens regulierend in die schulische Praxis ein - regulativ etwa durch Lehrpläne, Rundschreiben, Verordnungen, anleitend durch Fort- und Ausbildungsprogramme, Förderung von Projekten, diskursiv durch Wissensproduk- 
tion und -distribution in nahestehenden Institutionen oder Publikationsorganen. Dieser Beitrag fokussiert sich auf diese Interventionen, wobei das Bildungsdispositiv aus einer anordnenden Formation verschiedener behördlich-ministerieller, bildender, politischer und zivilgesellschaftlicher Institutionen und Instanzen besteht, deren Akteur/innen aus ihrer Vernetzung zueinander eine Diskursposition einnehmen, die es ermöglicht, Wissen zu produzieren und als legitimes Wissen zu positionieren.

- Diskursiv werden auf Basis von professionsspezifischem Wissen Leitbilder des idealen pädagogischen Handelns formiert, wodurch zugleich Bilder des/der professionellen Akteurs/in, d.h. einer beruflichen Identität (als Lehrer/in oder Schüler/in) konstituiert werden: „Diskurse erzeugen ,know how im Sinne der mehr oder weniger weit reichenden instrumentellen Befähigung zum Handeln hinsichtlich der jeweiligen Phänomenbereiche. Sie sind damit wiederum an der Herstellung von (bereichsspezifisch agierenden) sozialen Akteuren beteiligt" (Keller 2011, S. 237). Als konstitutive Bezugsnorm für professionelles Verhalten von Akteur/innen gewinnt Wissen eine handlungsanleitende Dimension: einerseits in gouvernementaler Perspektive, nämlich als Maßstab autonomer Optimierungsbestrebungen, andererseits, indem mit Konstruktionen beruflicher Identität Mechanismen der Ausschließung verbunden sind, durch Anrufung als professionelles Subjekt (,eine richtige Lehrerin würde so etwas nicht machen“) oder rechtliche Sanktionsmöglichkeiten (z. B. für Lehrpersonen standes- oder dienstrechtlich; für Schüler/innen in Haus- oder Prüfungsordnungen; vgl. Laclau und Mouffe 2001).

- Mit Rückgriff auf Alfred Schütz betont Keller (2008, S. 78) „die soziale Genese oder ,Konstruktion' der Deutungsschemata, die in Bewusstseinsprozessen zum Einsatz kommen und ein einfaches sinnliches, Erleben ' erst in reflektierte, sinnhafte ,Erfahrung 'verwandeln. "In der Schule leiten diskursive wie nicht-diskursive Praktiken Vorgänge der Subjektivierung an, indem sie ein spezifisches Interpretationsrepertoire bereitstellen, auf dessen Basis individuelle Sinn- und Deutungsstrukturen entstehen.

Im letzten Punkt deutet sich an, dass der Diskursbegriff der wissenssoziologischen Diskursanalyse (kurz: WDA) auch über eine materiale, praktische Dimension verfügt und so eine Unterscheidung zwischen diskursiven und nicht-diskursiven Praktiken zumeist nicht eindeutig getroffen werden kann. So gewinnt der Diskursbegriff Attraktivität für Fragestellungen, die sich unmittelbar auf Praxisfelder beziehen: Er verbindet die soziologische Frage nach Ordnungsstrukturen und Regeln von sozialen Feldern mit diskurstheoretischen Überlegungen ihrer Genealogie und Wirkung. Im Zuge schulischer Sozialisation kommt es zur Übernahme von Einstellungen, Normen und Werten; in diskurstheoretischer Perspektive wird dies (s. oben) als institutionelle Anleitung zu Praktiken und Positionen von Subjektivität gefasst. ${ }^{1}$ Die Frage nach Leitbildern idealen Lernens fragt damit zugleich, welche anthropologischen und gesellschaftlichen Ziel- und Wertvorstellungen einem Deutungsmuster zu

\footnotetext{
${ }^{1}$ Obgleich dies umstritten ist, plädiert Keller (2012, S. 72) für eine große Ähnlichkeit dieser begrifflichen Konzepte; hier soll dieser Argumentation gefolgt werden.
} 
Grunde liegen, d. h. wie eine inhaltliche Deutung über einen diskursiven Verlauf generiert wurde. und wie diese immanenten Normen, Ideologien und Weltbilder über die Organisation von Lernarrangements vermittelt werden.

Dieser Beitrag rekonstruiert den Prozess der Entstehung und Neuformierung der Aussage „Lernen soll Freude machen“ über die Instanz des Dispositivs. Keller (2011, S. 253) beschreibt Dispositive als „Infrastruktur der Diskursproduktion und Problembearbeitung “, die von einflussreichen Diskursen selbst erzeugt werden und über die Diskurse regulierend in die Praxis eingreifen. Foucault bezeichnet Dispositive als „Netz“ zwischen den heterogenen Elementen von Diskursen, Praxisfeldern und Akteur/innen, zwischen diskursiven und nicht-diskursiven Praktiken. Dispositive sind entsprechend als Korrelat ihrer Wirkung zu erkennen (vgl. Traue 2015). Entsprechend der materialen Fassung des Diskursbegriffs betont Keller (2008, S. 101) für die Analysepraxis, dass „Diskurs- und Dispositivperspektive kaum voneinander getrennt werden können “. Innerhalb eines Dispositivs können Prozesse der Verknappung, Neuformierung und Transformation diskursiver Äußerungen identifiziert werden; d. h. auch im Dispositiv werden aus verschiedenen Aussagen über das Lernen neue diskursive Formationen generiert; wie auch Diskurse sind Dispositive umkämpftes Terrain. Die Regelsysteme eines Dispositivs sind entscheidend dafür, wann Äußerungen an bestimmten Orten auftauchen und welche Stellung Äußerungen im Dispositiv einnehmen. Sie sind zugleich Reflexionsformen gesellschaftlicher Machtverhältnisse.

Als sondierendes Konzept der Analyse fungiert der Begriff des Deutungsmusters. Deutungsmuster stellen einen je spezifischen Teil des kollektiven Wissensvorrats dar, der zugleich auf die soziale, kulturelle und ökonomische Ebene der Gesellschaft verweist und mit ihr verwoben ist (Keller 2008). Die Annahme ist hierbei, dass die inhaltliche Reichweite von Deutungsmustern über den eigentlichen Gegenstand hinausgeht. Deutungsmuster von idealem Lernen üben nicht nur Einfluss auf Lernsettings, sondern generieren zugleich einen Teil der schulischen Alltagswelt, deren Ordnungen und Regeln Einfluss auf weitere Kontexte haben können. Eigene alltagspragmatische Deutungen und Sinnstrukturierungen können so mittels dieser Deutungsmuster stabilisiert werden. In einer Anpassung des Akteurskonzepts der WDA sollen in diesem Beitrag Deutungsmuster im Dispositiv bestimmt werden, indem die ursprüngliche Herkunft bestimmter Aussageformen aus Akteurskoalitionen im Diskurs zurückverfolgt wird, d.h. verknappte Aussagen des Dispositivs und ihre Bedeutung aus den ursprünglichen Aussagen rekonstruiert werden. Die Analyse kann als Deutungsmusteranalyse bezeichnet werden (vgl. dazu besonders Keller 2014). Das methodische Vorgehen der Analyse besteht in der Ermittlung von typischen Aussageformen, die ein fortlaufend auftretendes Muster von deutenden Elementen ergeben, von denen begründet angenommen werden kann, dass sie als strukturierendes Interpretationsschema zur Deutung von feldspezifischen Bedingungen und Modalitäten des idealen Lernens herangezogen werden können. In der Analyse wurden drei solche typischen Aussageformen ermittelt, die über jeweils ein bestimmtes Deutungselement verfügen. In Summe ergeben diese Elemente eine Deutungsfigur, ein Angebot, wie die Aussage „Lernen soll Spaß machen“ zu deuten ist. Der Mehrwert besteht laut Keller darin, dass die Deutungsmusteranalyse ein „,Brückenkonzept“ [darstellt], das gerade erlaubt, den Weg von der Diskursebene 
zur alltäglichen Deutungs- und Lebenspraxis zu gehen bzw. die Verflechtungen, Adaptionen, Aneignungen und Zurückweisungen im Deutungsmustergebrauch hier und da in den Blick zu nehmen " (Keller 2014, S. 155).

Mit diesen Überlegungen knüpft die WDA implizit an ideologiekritische Überlegungen von Gramsci an, der in Bildungsinstitutionen einen Ort der Durchsetzung einer hegemonialen Lebensweise und kulturellen Praxis durch alltägliche, aber ideologisch geformte Interaktionen erkennt (Merkens 2002). Dieser Beitrag argumentiert für eine Konvergenz dieser Zugänge und möchte Deutungsmuster nicht nur beschreiben, sondern ihnen aus einer pädagogischen Perspektive kritisch begegnen. Der Standpunkt dieses Beitrags kann so beschrieben werden, dass er als Ziele von Bildung (im Sinne der kritischen Pädagogik) den Gewinn von Autonomie und Reflexionsfähigkeit erkennt (vgl. Gruschka 1988; Sünker 2012). Die Funktion von Ideologie beschreibt Barrett (zit. nach Keller 2011, S. 159) als Mystifizierung sozialer Realitäten: ,discursive and significatory mechanisms that may occlude, legitimate, naturalize or universalize in a variety of different ways but can all be said to mystify “. Für Lernprozesse bedeutet dies, dass sie als ideologisch strukturiert gelten können, wenn sie ihre Konstitutionsbedingungen naturalisieren und ihre Erkenntnis und Reflexion erschweren, und dabei Normen, Werte und Einstellungen vermitteln, die im Sinne dominanter gesellschaftlicher Strukturen als funktional beschrieben werden können und diese legitimieren.

Die folgende Untersuchung fokussiert sich auf Dokumente des Bildungsdispositivs. Dazu zählen sowohl Dokumente der direkten Regulierung - Lehrpläne, Rundschreiben, Verordnungen - aber auch wissenschaftliche Dokumente, Dokumente aus der Aus- und Weiterbildung, Projektberichte, öffentliche Statements, sofern ein Naheverhältnis zum Dispositiv besteht. Im Zuge der Analyse wurden gesamt 233 solcher Dokumente gesichtet und ausgewertet Die folgende Auswertung bezieht sich auf die Aussage „Freude am Lernen“, einem von insgesamt drei zentralen Diskurssträngen, die ich in der Dissertation (Brandmayr 1026) analysiert habe.

\section{Zur diskursiven Formierung der Aussage „Spaß am Lernen“}

Überblicksmäßig betrachtet stellt die Forderung nach Spaß (oder Freude ${ }^{2}$ ) am Lernen im Diskurs heute eine Selbstverständlichkeit dar, die aber historisch betrachtet relativ neu ist. In Publikationen der 1970er und 1980er-Jahre wird Schule oftmals als Zwangsinstitution beschrieben. Vermerkt wird, dass Lernen prinzipiell Spaß machen könnte, das aber im Kontext von Schule nicht denkbar sei. In bildungspolitischen Forderungen dieser Zeit kommt die Forderung nach Freude und Spaß kaum vor. Im damaligen Parteiprogramm der Sozialdemokratischen Partei (1978, S. 32) Österreichs wird notiert: „Jedes Kind [...] hat ein Recht auf Erziehung ohne körperliche Züchtigung, auf Platz zum Spielen, auf eine menschenwürdige, gesundheitsfördernde Umwelt und auf eine humane Schule. " Der Begriff ,humane Schule“ impliziert nicht nur, dass die Schule der 1970er-Jahre in vielen Fällen nicht human war, sondern sich die Partei mit einer "humanen Schule“ schon zufrieden gegeben hätte

\footnotetext{
${ }^{2}$ In diesem Beitrag werden, wie auch im Diskurs, die Begriffe Spaß und Freude synonym verwendet.
} 
und eine Schule, die bei ihren Schüler/innen Freude hervorruft, gar nicht gefordert wurde.

Anfang der 1990er-Jahre äußern immer mehr Akteur/innen aus unterschiedlichen Perspektiven, dass Lernen Spaß machen sollte. Inzwischen hat diese Aussage eine hegemoniale Stellung im Bildungsdispositiv inne und wirkt sich dementsprechend auf die schulische Praxis aus. Im Folgenden sollen drei Deutungselemente der Aussage skizziert werden.

\subsection{Freude am Lernen als funktionales Element im Lernprozess}

Dieses Deutungselement basiert auf der anthropologischen Prämisse einer natürlich vorhandenen Freude am Lernen. Im Text der Expertenkommission zur Neugestaltung des Schulsystems wird der Wunsch zu lernen als kindlich-jugendlicher Erkenntnisdrang, von Freude begleitet, beschrieben: „Kindlich-jugendlicher Erkenntnisdrang, Wissbegierde, Lernfreude sind Grundlagen und Motor für Lernen, Bildung und Entwicklung - Schule sieht ihre Verantwortung, diese zu erhalten, zu nutzen und zu fördern " (Expertenkommission 2007, S. 10).

Dieses Deutungselement weist einen starken Bezug zu Aussagen neurodidaktischer Akteur/innen auf, welche Freude am Lernen als ein funktionales Element im Lernprozess deuten. Neurodidaktiker/innen betonen, dass Kinder bei erfolgreichem Lernen Freude empfinden, was sie über die Ausschüttung von Botenstoffen im Gehirn nachzuweisen versuchen: „Unter diesen Bedingungen wird im Gehirn immer auch eine Gruppe von Nervenzellen erregt und setzt [...] bestimmte Botenstoffe frei, die auch abgegeben werden, wenn Drogensüchtige Kokain und Heroin einnehmen. Das lässt erahnen, wie groß dieses Lustgefühl werden kann, das Kinder empfinden, wenn sie sich [...] auf den Weg machen, um die Welt zu entdecken" (Hüther 2006, S. 74). Schulkritik, wie sie von neurodidaktischen Akteur/innen besonders an repetitiven Lernformen und schulischen Disziplinierungsmaßnahmen geäußert wird, stützt sich dabei wesentlich auf ein Deutungsmuster, das Lust am Lernen als Möglichkeit zur Steigerung der Effizienz von Lernprozessen begreift und (gleichzeitig) diese Bedingungen in der Schule als nicht vorhanden erkennt. Eine typische Aussageform lautet entsprechend, dass Lernen ,gehirngerecht“ gestaltet werden soll.

Die Analyse zeigt, dass das Dispositiv diese Deutung übernommen hat. So wird im nationalen Bildungsbericht als Maßnahme für bessere Lesekompetenzen die Stärkung von Lesefreude genannt; der Begriff findet sich acht Mal. Als typische Aussage wird dabei vermerkt: „Auf der motivationalen Seite wird man zusätzlich versuchen, Freude am Lesen zu vermitteln, was etwa durch das ,Anlesen'von Büchern oder durch Bibliotheksbesuche mit der ganzen Klasse geschehen kann" (Schabmann et al. 2012, S. 39). Der Zusammenhang einer besseren Lesefähigkeit und Freude am Lesen wird dabei als Faktum anerkannt und nicht näher erläutert, wie auch die Zieldimension des schulisches Lernens, die mit Foucault (1994, S. 199) als ,erschöpfende Ausnutzung" kognitiver Ressourcen formuliert werden kann, nicht expliziert bzw. begründet wird. Freude erhält im Dispositiv jedoch die Stellung eines funktionalen Elements, durch das Lernprozesse effizienter gestaltet werden können, und ist diskursiv klar in diesem Deutungsmuster zu positionieren. 
Eine weitere Facette des Zusammenhangs von Freude und Effizienz erschließt sich durch folgendes Diskursfragment aus dem vom BMBF geförderten Projekt COOL: „Cooperatives Offenes Lernen bietet SchülerInnen die Möglichkeit, all ihre intellektuellen und emotionalen Fähigkeiten, ihre unterschiedlichen Begabungen, ihre Kreativität, ihre Neugier, ihre Spielfreude, ihre soziale Kompetenz in den Unterricht einzubringen. SchülerInnen arbeiten während der COOL-Phasen mehr als im traditionellen Unterricht [...]. Denn die Initiative geht von ihnen aus. Während die SchülerInnen arbeiten, können sich die LehrerInnen ganz bewusst den SchülerInnen widmen, die gefördert werden müssen, weil sie hoch begabt sind oder bestimmte Defizite haben " (Impulszentrum für cooperatives Lernen 2008, S. 16; Hervorh. M.B.). Es wird also direkt im Anschluss an die Aussage, dass Spielfreude und soziale Kompetenzen durch das COOL-Projekt zunehmen, auf die Effizienz des Projekts und seine Vereinbarkeit mit weiteren strategischen Zielen des Lerndispositivs hingewiesen. Dies ist vor dem Hintergrund brisant, dass sich das COOL-Projekt im theoretischen Rahmen von reformpädagogischen Ansätzen (Freinet und Parkhust) verortet. Akteur/innen, die sich an Diskussionen um Schulreform beteiligten und Veränderungen von Unterrichtsformen im Sinne reformpädagogischer oder sozialdemokratischer Ansätze (Arbeitsunterricht) forderten, äußerten sich zumeist kritisch gegen eine Ausrichtung der Schule am Leistungsprinzip; sie traten für Freude am Lernen ein, aber ohne damit weitere Ziele zu verfolgen (siehe auch Punkt b). Eine typische Aussage dieser Diskurskoalition lautete, dass eine zu starke Effizienzorientierung sich nicht mit einem (selbst so verstandenen) Leitbild von progressivem Unterricht (mit Zielen wie Freiheit, Demokratisierung, und auch Lernfreude) vereinbaren lässt, sondern vielmehr Unmündigkeit und Unterordnung bewirke (vgl. Beck 1974). Festzustellen ist daher ein Moment der diskursiven Verknappung, wodurch eine ursprüngliche Widersprüchlichkeit in Deutungen von idealen Lernmodalitäten nun nicht mehr kenntlich wird. ${ }^{3}$ Diese Projekte bieten jedoch an vielen Stellen Identifikationsangebote für Akteur/innen der politisch links stehenden Diskurskoalition, welche durchaus ähnliche Formulierungen verwenden, obwohl Freude in dieser Deutung nicht primär zur Humanisierung, sondern zur Effizienzsteigerung des Unterrichts beitragen soll.

\subsection{Freude am Lernen als Freude an der Leistung}

Eine besondere Bedeutung erlangt die Forderung nach Freude am Lernen im Hinblick auf die Leistungsbeurteilung. Denn wie dies etwa die ehemalige österreichische Unterrichtsministerin Claudia Schmidt (2008, S. 14) ganz offen formuliert, wird vom Lerndispositiv eine zunehmende Leistungsorientierung angestrebt: „,Meine Traumschule ist eine Leistungsschule, in der verstärkt auf Begabungen und Talente eingegangen wird. "Um dies mit Freude am Lernen zu verbinden, formulieren Benischek und Beer (2011, S. 19): „Die Postulierung eines produktiven Leistungsbegriffs

\footnotetext{
3 Diese Aussage findet sich etwa auch im Projekt KLIBO: Dies intendiert ebenso einen motivierenden, aktivierenden und freudvollen Unterricht durch Individualisierung und offene Lernformen, argumentiert aber zugleich mit Effizienz und besseren Lernerfolgen (vgl. BMBF 2012b).
} 
von einer Leistungsbürde hin zur Freude an individuell erbrachter Leistung ist von zentraler Bedeutung “. Wie wird diese Verknüpfung unternommen?

Reformpädagogische und sozialdemokratische Konzepte deuten freiwillig erbrachte Lernprodukte, die ohne Zeit- und Notendruck angefertigt werden können, als Möglichkeit, freudvoll Leistung zu erbringen. Diese Konzepte wurden in strikter Opposition zur schulischen Praxis konventioneller Prüfungssituationen formuliert, denn ,wie soll Lernen Freude machen, wenn die Angst vor der Prüfung ständig im Nacken sitzt?" (Kössmeier 2007, S. 3). Ihr Ziel sollte einerseits sein, Bedingungen von freudvollem und emanzipativem Lernen sicherzustellen und Lernprozesse ohne Druck zu begleiten; andererseits war Lernen in dieser Deutung an Ziele von Autonomie und Freiheit gebunden. Lernleistungen wurden als Dokumentation des individuellen Fortschritts erachtet, mit der Möglichkeit, sich in seiner eigenen Tätigkeit zu erkennen und darin zu wachsen. Die Vorstellung, die Arbeit als Verwirklichung bzw. Ausdruck der menschlichen Persönlichkeit begreift, lässt sich bei sozialdemokratischen Schulakteur/innen bis zu Marx' (1968, S. 517) früher Theorie zur Entfremdung zurückverfolgen, der dem Produkt industrieller Arbeitsverhältnisse, als entfremdete Arbeit, das Konzept erfüllender Arbeit gegenüberstellt, in der sich der Arbeiter ,nicht nur wie im Bewusstsein intellektuell, sondern werktätig, wirklich verdoppelt, und sich selber daher in einer von ihm geschaffenen Welt anschaut" d. h. erst zum Menschen wird. Sie findet sich wieder im Konzept des Arbeitsunterrichts wie auch in handlungsorientierten Zugängen, z. B. jenem von Dewey, der manuelle Arbeit als Möglichkeit zu Autonomiegewinn interpretiert.

Eine bildungsökonomisch argumentierende Akteurskoalition vertritt eine Deutung von idealem Lernen, die Lernleistungen als immer gleichzeitig subjektiv und gesamtwirtschaftlich bedeutungsvoll sieht. Eine Prämisse wird dabei typisch so formuliert: „Bildung ist Entwicklung und damit Chance für jede Person [...]. Bildung führt somit zu persönlichem Glück, sozialem Wohlstand und sich." (Monika KircherKohl, zit. nach: Industriellenvereinigung 2009, S. 11). Die gesellschaftliche Bestimmung von Lernzielen tritt hinter die subjektive Relevanz, weil dem Subjekt zugleich die materielle Absicherung seiner Existenz eigenverantwortlich übertragen wird. Wenn gefordert wird, dass Unterricht ,die Prinzipien der Selbststeuerung und Eigenverantwortung zu Grunde“ (Industriellenvereinigung 2014, S. 16) liegen, dann auch, weil Individuen über die Relevanz von Inhalten im Hinblick auf ihr späteres Fortkommen selbst entscheiden müssen. Diese Deutung abstrahiert die gesellschaftliche Aufforderung zu lernen und zu leisten; sie definieren sich über den subjektiven Erfolg.

Im Lerndispositiv erfährt der Leistungsbegriff aus der Verschränkung dieser Positionen eine Neubestimmung. Eine typische Aussage dazu findet sich in den Leitlinien zu Schulqualität Allgemeinbildung: „Last but not least: Leistung. Leistung wird gefordert, ist wichtig und stärkt das Selbstwertgefühl (,Es war ein hartes Stück Arbeit, aber ich hab's geschafft!') und steigert die Bereitschaft, sich neuen Herausforderungen zu stellen, was zu neuerlichem Wissens- bzw. Kompetenzzuwachs führt. Positive Lernergebnisse, erreichte Ziele setzen eine Motivationsspirale in Gang, sie können uns im wahrsten Sinne des Wortes beflügeln. Leistung und Freude am Lernen sind also kein Widerspruch, ganz im Gegenteil!“ (BMBF 2012a, S. 3). Hier lässt sich eine Deutung nachzeichnen, wonach Freude am Lernen als Freude an der Leistung 
zu verstehen ist, im Sinne einer Freude am konkreten Produkt. Dabei soll in bestimmtem Rahmen freiwillig und selbstbestimmt geleistet werden. Der Begriff wird weder auf schulisch vorgegebene Leistung reduziert noch als fokussiertes Lernprodukt gefasst, sondern bezieht sich auf alle Formen produktiver Tätigkeiten in der Schule. Lernprodukte materialisieren sich als konkretes Produkt jenseits eines gesellschaftlichen Maßstabs, was als erfolgreiches Lernergebnis zu gelten hat. Als subjektives Produkt kann jede messbare, materialisierte Lernleistung als potentiell freudvoll empfunden werden.

Mögliche negative Effekte des Leistungsprinzips, wie Leistungsdruck, eine Konkurrenz zwischen Lernenden, die Konfrontation mit Erwartungen und der Zwang, sichtbaren Output aus Lernhandlungen zu generieren, bleiben zwar bestehen; aber sie sind in dieser Deutung von Leistung diskursiv vom gesellschaftlichen Leistungsprinzip getrennt. Ein Hinweis auf Formen von Subjektivität, die als Nebeneffekte von Leistungsdenken entstehen können - beispielsweise ein Konkurrenzdenken oder geringere Bereitschaft zu solidarischem Verhalten - kann sich in kein diskursives Verhältnis mehr zur Deutung von Leistung im Dispositiv setzen, die in der Äußerung kulminiert, dass „,dokumentierte individuelle Leistungserfolge [...] die Freude am Leisten aller Kinder [erhöhen]" (Benischek und Beer 2011, S. 19). Das Bildungsdispositiv hat in der Akzentuierung der individuellen Leistungsfreude die gesellschaftliche Dimension von Leistung und Leistungsbereitschaft (und damit sozialdemokratische Einwände) diskursiv verknappt, ohne dabei den (ökonomisch höchst relevanten) Charakter der Messbarkeit und der Outputorientierung von Lernleistungen zu beschneiden. Das Erbringen von Lernleistungen erfolgt auf individuelle Aufforderung in individuellem Rahmen; die Unsichtbarkeit der normativen Aufforderung macht es möglich, in der Schule Freude am leistungsorientierten Lernen zu empfinden.

\subsection{Freude als Lernziel}

Äußerungen, die Freude als Lerngegenstand und Lernziel formulieren, verweisen nahezu ausschließlich auf bildungsökonomische Deutungselemente. Einerseits findet sich eine Argumentation, die Weiterbildungsmaßnahmen als in der heutigen Ökonomie notwendig erachtet und in der sich im Kontext einer Funktionalitätsannahme (siehe Deutungsmuster a) eine Engführung der Begriffe Freude am Lernen, Erfolg und den Verläufen beruflicher Biografien zeigt: „Die Freude am Lernen (in der Schule) müsste wachsen, denn wer sein Leben erfolgreich gestalten möchte, muss lernen können und es auch wollen (Müller 2006, S. 7). Die Notwendigkeit dieser Forderung zeigt sich ebenso darin, dass nur mehr ein Bruchteil der heute Vierzigjährigen in ihren erlernten Berufen tätig ist, neue Arbeitsfelder kommen hinzu und vertraute verschwinden" (Benischek und Beer 2011, S. 5).

Ein zweites Deutungselement begründet Freude am Lernen im Sinn eines kognitiven Kapitalismus, in dem Persönlichkeitsmerkmale eine Ressource darstellen. Dieses Deutungselement lässt sich bis an die Anfänge des Diskurses um Lebenslanges Lernen in England zurückverfolgen (vgl. Landry und Bianchini 1995). Im deutschsprachigen Raum nachhaltige diskursive Wirkung entfalten vor allem Beiträge von Peter Hartz, der in Deutschland den Arbeitsmarkt reformiert und Sozialbezüge an 
Qualifizierungsmaßnahmen geknüpft hat. In der neuen Ökonomie, so schreibt Hartz (zit. nach Haug 2003, S. 608), ist der ganze Mensch gefragt, mit seinen individuellen Möglichkeiten, seiner Offenheit, seinem Talent und seiner Leidenschaft, zu lernen, zu entdecken, etwas zu entwickeln und weiterzugeben. Es lebe der kreative Unterschied. Wir lassen den Taylorismus hinter uns. "Für die Arbeitsplätze von morgen werden sowohl kognitive Ressourcen wie Kreativität, Kompetenzen und Soft Skills, aber auch emotionale Dispositionen, die zu einer neuen Identifikation mit dem Beruf führen, wichtig. Diese gesteigerten Anforderungen bedeuten, dass Bildungssysteme nun den ,ganzen Menschen“ in den Blick nehmen müssen.

Der Einfluss dieses bildungsökonomischen Diskurses lässt sich im Dispositiv beispielsweise daran festmachen, dass persönlichen Maßstäben der Lebensführung eine Bedeutung als Lerngegenstand zukommt. So zeigt sich im Grundsatzpapier zum Ausbau der Berufsorientierung und Bildungsberatung an Schulen eine Deutung, die (berufliches) Lernen als Weiterbildungs- und Qualifizierungsmaßnahme für den ganzen Menschen versteht: „Berufsorientierung heißt Lebensorientierung und ist somit als Prozess zu verstehen, der früh beginnt und über die Schulzeit hinausgeht. Bereits die derzeitige Situation in der Arbeitswelt, mit sich stark verändernden Qualifikationsanforderungen und Berufsprofilen, und die zu erwartende weitere Beschleunigung dieser Entwicklungen in der Zukunft, erfordern ständige Weiterbildung und wiederholte Neuorientierungen. Dementsprechend wird die Schaffung eines lebensbegleitenden Systems von Information, Beratung und Orientierung (Lifelong Guidance) als wichtige gemeinsame europäische bildungspolitische Zielsetzung gesehen “ (BMUKK 2010, S. 1). Der Begriff „Lifelong Guidance“ ist hier vielsagend, da er das institutionelle Interesse an der Steuerung personaler Orientierungen markiert. Er beschreibt eine Deutung, wonach spezifische Qualifizierungsmaßnahmen mit Neu- oder Umorientierung von Aspekten der Lebensführung an strategischen Zielen des Marktes einhergehen.

In einem Interview vermerkt Claudia Schmidt (2008, S. 14) auf die Frage, welche Fähigkeiten aus wirtschaftlicher Sicht in der Schule gestärkt werden sollten: „Die Schule nimmt immer mehr die Rolle eines Lebensraumes ein, der eine Vorbereitung für zukünftige Lebensräume darstellen muss. Wir brauchen Menschen mit Eigenschaften. “ Die Forderung, ,Eigenschaften“ in Hinblick auf spätere, d. h. ökonomische, Kontexte auszubilden, kann für die Schulpraxis als Steuerung von Subjektivierungsprozessen interpretiert werden, um eine Konvergenz zwischen der Ausprägung von Persönlichkeitsmerkmalen und ihrem Bedarf im modernen Kapitalismus zu erreichen. Wenn das Ziel im Induzieren von Freude am Lernen besteht, wird Freude am Lernen dann erreicht, wenn eine Identifikation mit neuen Zieldimensionen von Lebensführung, also eine neue „Lebensorientierung“ im Prozess von Schule oder Weiterbildung vollzogen ist. Freude wird so selbst zum Gegenstand der Umorientierung: Die Institution Schule verfügt über eine Deutung, was Freude ist, wann Freude empfunden werden kann, und setzt sich zum Ziel, diese Deutung zu vermitteln. Freude in den ,richtigen“ Kontexten zu empfinden, kann demnach eine solche Eigenschaft sein, die „man braucht“.

Diese Interpretation unterstreicht folgendes Diskursfragment mit dem Titel „Schule bereitet auf ein glückliches Leben vor". Darin heißt es: „Die Investition in qualitätsvolle Bildung und Erziehung und die Förderung von Resilienz und emo- 
tionaler Intelligenz ermöglicht es Menschen, ein gesundes und produktives Leben zu führen. Daher sind sich Pädagoginnen und Pädagogen an guten Schulen ihrer Verantwortung bewusst, was und wie sie dazu beitragen, dass junge Menschen ein glückliches Leben führen können" (ZLS 2015, S. 8). Die Formulierung, dass „Resilienz und emotionale Intelligenz“ ein „glückliches und produktives Leben“ ermöglichen, verweist schon auf einige Aspekte einer institutionellen Deutung von Lebensführung, die zu Freude führt. Schule wird zum Lebensraum, in dem Erfahrungen gemacht werden, die die Übernahme spezifischer Einstellungen forcieren. Emotionale Bezüge wie Freude werden der Norm gegenwärtiger Lebensführung unterworfen; die Institution formt ihren Charakter und setzt sie in Bezug zu spezifischen Anforderungen, konkret ökonomischer Handlungsfähigkeit.

\section{Fazit}

Diese bislang ausgeführten Überlegungen sollen zeigen, wie gesellschaftliche Diskurse über Leitbilder von idealem Lernen Einfluss auf die Praxis schulischer Lernprozesse nehmen. Am Beispiel der Aussage „Freude am Lernen“ lässt sich deutlich erkennen, dass es im Dispositiv zu Verknappungs- und Neuformierungsprozessen kommt, wodurch die Aussage sowohl inhaltlich verfremdet, aber auch naturalisiert wird. Sie bietet sich als Identifikationspunkt für verschiedene Akteur/innen an, obgleich sich ihre Bedeutung hinsichtlich ihrer ursprünglichen Verwendungsweise im Kontext spezifischer Akteursgruppen gewandelt hat. Als verfremdete Aussage transportiert sie Normen, Werte und Einstellungen des Dispositivs, die erst durch diese Analyse inhaltlich bestimmbar wurden. Zu thematisieren wäre auch, ob und wenn ja wie der Aussage eine ideologische Funktion in der schulischen Praxis zukommt - eine Frage, die hier offen bleiben muss.

Der Mehrwert der Analyse besteht nun darin, dass eine diskursive Betrachtung des Lernens einen Beitrag zur Diskussion leisten kann, nach welchen Prinzipien und Regeln schulisches Lernen tatsächlich organisiert wird. Sie kann einen Denkanstoß geben, um in der Diskussion über einen pädagogischen Begriff des Lernens eine neue Perspektive zu etablieren, die sich an einer empirischen Ebene orientiert. In dieser Perspektive können aber auch Fragen des ,,heimlichen Lehrplans“ (Zinnecker 1975) oder der gesellschaftlichen Funktion von Schule neu gestellt werden.

Open access funding provided by University of Innsbruck and Medical University of Innsbruck.

Open Access. Dieser Artikel wird unter der Creative Commons Namensnennung 4.0 International Lizenz (http://creativecommons.org/licenses/by/4.0/deed.de) veröffentlicht, welche die Nutzung, Vervielfältigung, Bearbeitung, Verbreitung und Wiedergabe in jeglichem Medium und Format erlaubt, sofern Sie den/die ursprünglichen Autor(en) und die Quelle ordnungsgemäß nennen, einen Link zur Creative Commons Lizenz beifügen und angeben, ob Änderungen vorgenommen wurden. 


\section{Literatur}

Beck, J. (1974). Lernen in der Klassenschule. Hamburg: Rowohlt.

Beer, R., \& Benischek, I. (2011). Aspekte kompetenzorientierten Lernens und Lehrens. In BIFIE (Hrsg.), Kompetenzorientierter Unterricht in Theorie und Praxis (S. 5-29). Graz: Leykam.

BMUKK (2010). Grundsatzpapier Ausbau der Berufsorientierung und Bildungsberatung. https://www. bmbf.gv.at/schulen/bo/ibobbgrundsatzpapiernov2010o_21088.pdf?4jzz6d. Zugegriffen: 1. März 2016

BMBF (2012a). Schulqualität Allgemeinbildung. Über das Lernen. www.sqa.at/course/view.php?id=35. Zugegriffen: 1. März 2016

BMBF (2012b). Rundschreiben 17 NEU - Information, Beratung und Orientierung für Bildung und Beruf (IBOBB) - Grundsätzliches und wichtige Voraussetzungen. https://www.bmbf.gv.at/2012_17_23228. pdf?4dtiae. Zugegriffen: 1. März 2016

Brügelmann, H. (2008). Wie verbreitet ist offener Unterricht? In H. Brügelmann, \& E. Brinkmann (Hrsg.), Öffnung des Anfangsunterrichts. Theoretische Prinzipien, Unterrichtspraktische Ideen und empirische Befunde. Siegen: Arbeitsgruppe Primarstufe/Universität.

Expertenkommission zur Zukunft der Bildung (2007). Erster Zwischenbericht. https://www.bmbf.gv.at/ schulen/bw/nms/ek_zwb_01_15690.pdf?4zsea5. Zugegriffen: 1. März 2016

Foucault, M. (1994). Überwachen und Strafen. Frankfurt am Main: Suhrkamp.

Grotlüschen, A. (2014). Neo-subjektwissenschaftliche Lesart einer scheinbar vertrauten Lerntheorie. In P. Faulstich (Hrsg.), Lerndebatten (S. 225-258). Bielefeld: Transcript.

Gruschka, A. (1988). Negative Pädagogik. Einführung in die Pädagogik mit Kritischer Theorie. Wetzlar: Büchse der Pandora.

Gruschka, A. (2011). Verstehen lehren. Ein Plädoyer für guten Unterricht. Marburg: Reclam.

Haug, F. (2003). Schaffen wir einen neuen Menschentyp. Das Argument, 252, 606-626.

Holzkamp, K. (1993). Lernen. Subjektwissenschaftliche Grundierungen. Frankfurt New York: Campus.

Holzkamp, K. (1992). Die Fiktion administrativer Planbarkeit schulischer Lernprozesse. In K.-H. Braun, \& K. Wetzel (Hrsg.), Lernwidersprüche und pädagogisches Handeln. Marburg: Arbeit und Gesellschaft.

Hüther, G. (2006). Wie lernen Kinder? Voraussetzungen für gelingende Bildungsprozesse aus neurobiologischer Sicht. In R. Caspary (Hrsg.), Lernen und Gehirn . Der Weg zu einer neuen Pädagogik (S. 70-84). Freiburg: Herder.

Impulszentrum für Cooperatives Offenes Lernen (2008). COOL - Cooperatives Offenes Lernen. http:// www.abc.berufsbildendeschulen.at/upload/1373_Cool_Booklet_Letztversion_lr_081014.pdf. Zugegriffen: 1. März 2016

Industriellenvereinigung (2009). Schule 2020. Lernen - Wachstum - Wohlstand. www.iv-net.at/iv-all/ publikationen/file_488.pdf. Zugegriffen: 1. März 2016

Industriellenvereinigung (2014). Beste Bildung für Österreichs Zukunft. www.iv-net.at/d4300/beste_ bildung.pdf. Zugegriffen: 1. März 2016

Keller, R. (2014). Wissenssoziologische Diskursforschung und Deutungsmusteranalyse. In C. Behnke, D. Lengersdorf, \& S. Scholz (Hrsg.), Wissen - Methode - Geschlecht (S. 143-159). Wiesbaden:: Springer.

Keller, R. (2012). Der menschliche Faktor. In R. Keller, W. Schneider, \& W. Viehöver (Hrsg.), Diskurs Macht - Subjekt (S. 69-109). Wiesbaden: Springer.

Keller, R. (2011). Wissenssoziologische Diskursanalyse. Grundlegung eines Forschungsprogramms. Wiesbaden: Springer.

Keller, R. (2008). Diskurse und Dispositive analysieren: Die wissenssoziologische Diskursanalyse als Beitrag zu einer wissensanalytischen Profilierung der Diskursforschung. Historical Social Research, 33(1), 73-107.

Kössmeier, E. (2007). Individualisierung. Mein persönlicher und fachlicher Bezug zum Thema. Rotstift, $88,3-4$

Laclau, E., \& Mouffe, C. (2001). Hegemony and Socialist Strategy. London: Verso.

Landry, C., \& Bianchini, F. (1995). The Creative City. London: Demos.

Ludwig, J. (2005). Bildung und expansives Lernen. Hessische Blätter für Volksbildung: Zeitschrift für Erwachsenenbildung in Deutschland, 4, 328-336.

Marx, K. (1968). Die entfremdete Arbeit . Marx-Engels-Werke Ergänzungsband I. Berlin: Dietz.

Merkens, A. (2002). Ideologie, Kritik und Bildung. Das Argument, 246, 339-353.

Müller, A. (2006). Eigentlich wäre Lernen geil. Wie Schule (auch) sein kann: alles ausser gewöhnlich. Bern: Hep Verlag. 
Patzner, G. (2007). Offener Unterricht“ - ein neoliberales Führungsinstrument. In M. Heinrich, \& U. PrexlKrausz (Hrsg.), Eigene Lernwege - Quo vadis? (S. 59-78). Wien Münster: LIT.

Schmidt, C. (2008). Schule im Aufbruch. ILS-Mail 1/2008, 14-15. http://www.uibk.ac.at/ils/ilsmail/pdf_ ils_mail/schule_im_umbruch_online.pdf. Zugegriffen: 1. Feb 2016

Sozialdemokratische Partei Österreichs (1978). Das neue Programm. http://www.renner-institut.at/ uploads/media/1978_SPOE-Parteiprogramm_11_Auflage_1987_01.pdf. Zugegriffen: 1. März 2016

Schabmann, A., Landerl, K., Bruneforth, M., \& Schmidt, B. M. (2012). Lesekompetenz, Leseunterricht und Leseförderung im österreichischen Schulsystem. In B. Herzog-Punzenberger (Hrsg.), Fokussierte Analysen bildungspolitischer Schwerpunktthemen Nationaler Bildungsbericht Österreich 2012, (Bd. 2, S. 17-72). Graz: Leykam.

Schrittesser, I. (2012). Lernen pädagogisch gewendet. Annäherungsversuche mit Nebenwirkungen. In I. Schrittesser et al. (Hrsg.), Innovative Learning Environments - Fallstudien zu pädagogischen Innovationsprozessen (S. 95-122). Wien: Facultas/WUV.

Sünker, H. (2012). Historisch-materialistische Ansätze in Bildungsforschung und Pädagogik. In U. Bauer, U. Bittlingmayer, \& A. Scherr (Hrsg.), Handbuch Bildungs- und Erziehungssoziologie (S. 335-351). Wiesbaden: Springer.

Traue, B. (2015). Dispositiv. In D. Wrana et al. (Hrsg.), DiskursNetz - Wörterbuch der interdisziplinären Diskursforschung (S. 124-125). Frankfurt: Suhrkamp.

Zinnecker, J. (1975). Der heimliche Lehrplan. Weinheim und Basel: Beltz.

ZLS - Zentrum für lernende Schulen (2015). Gute Schule - Neue Mittelschule. http://www.nmsvernetzung. at $/$ mod/glossary/view.php?id=2473\&mode=entry\&hook=4304. Zugegriffen: 1 . März .2016

Mag. Michael Brandmayr Senior Lecturer 